Case Reports

\title{
A Case of Leptospira Ards- "Obsession with COVID-19 Leading to Delayed Diagnosis of Common Disease
}

\author{
Prakash Tendulkar, Divanshee Sharma, Mukesh Bairwa and Ravi Kant \\ Department of General Medicine, All India Institute of Medical Sciences, Rishikesh, India
}

\author{
Article history \\ Received: $30-12-2020$ \\ Revised: 28-02-2021 \\ Accepted: 25-03-2021 \\ Corresponding Author: \\ Divanshee Sharma \\ Department of General \\ Medicine, All India Institute of \\ Medical Sciences, Rishikesh, \\ India \\ Email: dr.divansheesharma89@gmail.com
}

\section{Introduction}

\section{Background}

In leptospirosis, hepatorenal insufficiency with severe respiratory distress is a commonly encountered clinical condition. In many patients with multiorgan dysfunction aetiology it is not easily identifiable specially when the disease progresses very rapidly in the current situation of COVID-19 19 pandemic.

\section{Case History}

A 23-year-old male student belonging to low socioeconomic status, who was smoker (6 Pack years) and occasional alcoholic and had no previous comorbidity. He presented to us with complains of fever for 1 week, it was mild in intensity and got resolved with antipyretics. It was associated with shortness of breath for 4 days which was gradually progressive and associated with mild yellowish expectoration. He also complained of loose stools for 4 days. It was watery in consistency with frequency of 5-6 episodes/day. Since last few weeks he was helping his family in farming activity like irrigation, as due to ongoing covid pandemic all educational institutions were closed. His father also complained of low-grade fever for 5 days which got resolved with over-the-counter antipyretics. On examination, his saturation was $62 \%$ on room air. He was started on moist oxygen support of 10liter/min with face mask and saturation improved to 95\%. Lung USG s/o bilateral B-profile. In view of ongoing COVID-19 pandemic, differential of COVID pneumonia was made and started on injection dexamethasone and other supportive treatment. Chest $\mathrm{x}$ ray was done s/o b/l diffuse infiltrates (Fig. 1). Blood sample for complete blood count, renal function test and liver function test were sent. COVID-19 RT-PCR sample was sent which came out to be negative. Routine blood investigations were sent and trend is shown in Table 1. In view of high clinical suspicion, repeat COVID-19 RTPCR and COVID-19 serology sample were sent which came out to be negative. Over the time his clinical condition worsened in form of tachypnoea and type-1 respiratory failure as suggested by sequential arterial blood gas analysis. He was started on NIV with Fi02 of $60 \%$, PEEP of 8 and Psupp of 14. He improved with NIV and tachypnoea resolved and was maintaining saturation of above $90 \%$. considering the rapid progression of respiratory complains repeat Covid RT-PCR and serology was sent. Total of 4 Covid RT-PCR and twice covid serology came out to be normal. HRCT thorax was done s/o diffuse ground glass attenuation in bilateral lung parenchyma, more in bilateral lower lobes-Features s/o ARDS/non-cardiogenic pulmonary oedema (Fig. 2). His blood reports s/o Direct hyperbilirubinemia, serum Leptospira Ig M sample was sent and he was started on Inj Doxycycline. Serum sample for Hep A, B, C and E 
were also sent which came out to be non-reactive. USG whole abdomen was done which was suggestive of hyperechoic liver. meanwhile patient condition deteriorated and elective intubation was done as he was not tolerating NIV. Lung protective ventilation was done for ARDS (TV $=4-6 \mathrm{ml} / \mathrm{kg}$ and PEEP of 12). He underwent Bronchoscopy with BAL and sample sent for bacterial, mycobacterial, viral and fungal cultures which later came out to be sterile. His clinical condition deteriorated very rapidly and was not maintaining saturation even with Fio2 of $100 \%$. But despite of bestefforts patient expired during hospital stay. Later his Leptospira IgM enzyme immunoassay test came out to be positive, value more than twice the normal range $(2.13$ index) (Normal- <0.9) and diagnosis of Leptospira induced ARDS was confirmed.

Table 1: Laboratory investigations

\begin{tabular}{lllll}
\hline Investigation & $03 / 10$ & $06 / 10$ & $08 / 10$ & $10 / 10$ \\
\hline Hb & 14.1 & 14.29 & 13.7 & 11.68 \\
TLC & 16.8 & 13.94 & 23.54 & 24.3 \\
DLC (N/L/M/E) & $87 / 4 / 6 / 0$ & $81 / 7 / 10 / 0$ & $85 / 4 / 6 / 2$ & $83 / 5 / 10 / 0$ \\
PLT (Lakh) & 3.59 & 3.87 & 5.31 & 5.42 \\
Urea & 87.1 & 83.2 & 52.1 & 37.3 \\
Creatinine & 3.29 & 1.29 & 0.77 & 0.79 \\
Na/K/Ca & $137 / 3.8 / 10.34$ & $145 / 4.5 / 9 / 96$ & $142 / 4.2 / 9.25$ & $129 / 4.1 / 9.24$ \\
T Bilirubin & 6.61 & 11.24 & 16.7 & 20.95 \\
D. Bilirubin & 5.0 & 8.28 & 11.79 & 15.12 \\
SGPT & 148 & 244 & 253 & 320 \\
SGOT & 127 & 411 & 401 & 427 \\
ALP & 800 & 1080 & 992 & 1269 \\
GGT & 386 & 176 & 275 & 268 \\
S. Albumin & 4.43 & 3.71 & 3.72 & 3.45 \\
INR & 1.16 & 1.15 & 1.31 & \\
Hep-B, Hep-C, HIV & Non-reactive & & & Non- Reactive \\
Hep-A, Hep-E & & & & \\
Amylase & 71.6 & & & \\
Lipase & 30.8 & & & \\
\hline
\end{tabular}

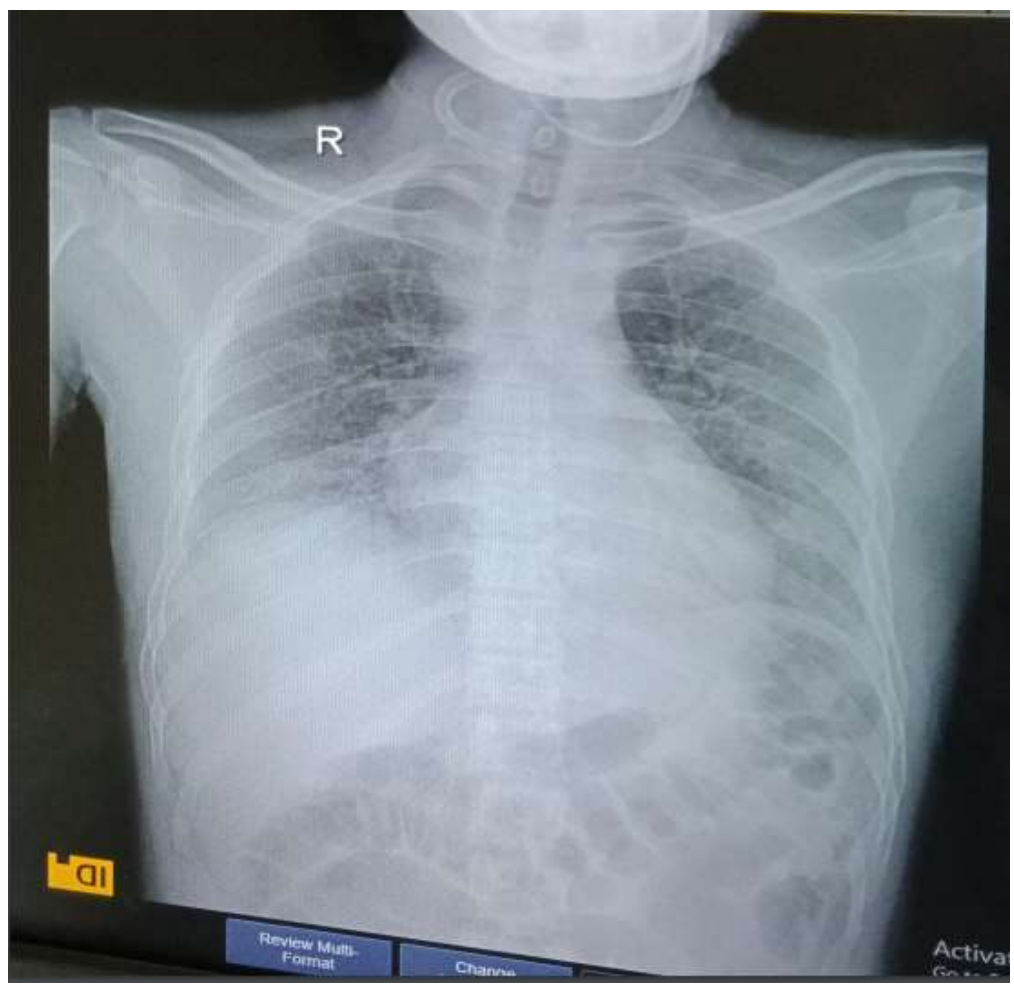

Fig. 1: Chest $\mathrm{X}$-ray s/o B/L diffuse infiltrates 


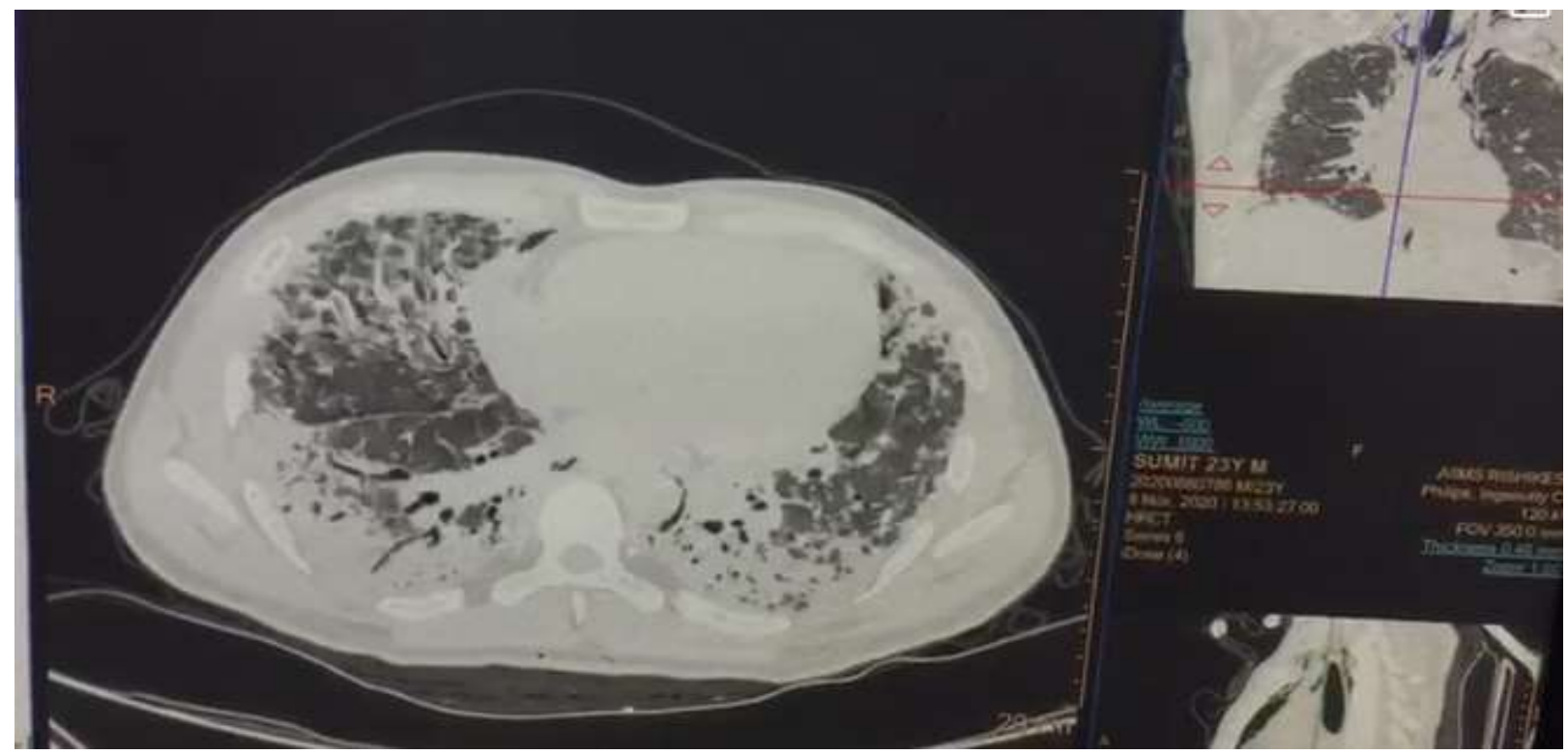

Fig. 2: Diffuse ground glass attenuation in bilateral lung parenchyma, more in bilateral lower lobes-Features s/o ARDS/noncardiogenic pulmonary oedema

\section{Discussion}

According to data released on 21st 2021 from WHO total 110,974,862 confirmed cases are diagnosed till now all over the world with total death attributed to COVID19 is 2,460,792. Also, data released from Ministry of Health and Family Welfare India (MoHFW) on 19th feb 2021 total 10,667,741 confirmed positive cases were diagnosed in India so far with death attributed due to COVID-19 is $1.42 \%$. According to same source, In the state Uttarakhand Himalayan region total of 94850 confirmed cases are diagnosed with Covid with total death of 1686 (PPCL, 2021). Out of these, 2396 got admitted at our tertiary care hospital with total death of 487 patients. Leptospirosis is a zoonotic disease which is more common in coastal regions of India and show seasonal variation as it more common in rainy season. Sporadic cases may occur throughout the year (Mehta et al., 2019). In Uttarakhand Himalayan region prevalence of leptospirosis is only $0.3 \%$.

ARDS is a common complication and the leading cause of covid related mortality. Cytokine Release Syndrome (CRS) or cytokine storm is typically associated with COVID-19 and marked with the overproduction of pro-inflammatory cytokines. The high morbidity and mortality are seen in COVID-19 can be attributed to autoimmune injury to lungs due to release of proinflammatory cytokines. So anti-inflammatory drugs like corticosteroid is commonly used to decrease the lung injury by cytokines in Acute Respiratory Distress Syndrome (ARDS) (Roshanravan et al., 2020; Garcia et al., 2000). In our case patient gradually developed severe ARDS, so he was started on injection dexamethasone in view of current covid pandemic. Despite classic inaugural clinical presentation, delayed diagnosis of Leptospira was made, considering ARDS due to current covid-19 pandemic. Also, prevalence of the disease is very low in Uttarakhand Himalayan region which make the diagnosis even more challenging. Although, frequent but pulmonary symptoms are usually mild in leptospirosis. in our case patient had AKI initially which got resolved over the hospital course. But his hepatic involvement got worsened over the course which gave us the clue to search for alternative diagnosis. He was also a smoker which is a risk factor for Leptospira induced ARDS (Gulati and Gulati, 2012). Also, patient condition deteriorated very rapidly and progressed to severe ARDS leading to mortality despite best of efforts.

\section{Conclusion}

Leptospirosis symptoms can range from mild muscle pains, headache and fever to severe forms including jaundice and renal failure. Severe ARDS is rare complication of leptospirosis and in background of COVID-19 pandemic diagnosis can be missed very often. Clinician should be aware of these rare complications and many a times these are missed due to COVID-19 pandemic.

\section{Author's Contributions}

Prakash Tendulkar: Manuscript writing, data collection, case report.

Divanshee Sharma: Literature search, manuscript writing and editing. 
Mukesh Bairwa and Ravi Kant: Manuscript review and editing.

\section{Ethics}

This article is original and contains unpublished material. The corresponding author confirms that all of the other authors have read and approved the manuscript and no ethical issues involved.

\section{References}

Clavel, M., Lhéritier, G., Weinbreck, N., Guerlin, A., Dugard, A., Denes, E., \& Vignon, P. (2010). Leptospirosis: An unusual cause of ARDS. Critical Care Research and Practice, 2010. https://doi.org/10.1155/2010/408365

Garcia, M. M., de Diego Damia, A., Villanueva, R. M., \& Hontagas, J. L. (2000). Pulmonary involvement in leptospirosis. European Journal of Clinical Microbiology and Infectious Diseases, 19(6), 471-474. https://doi.org/10.1007/s100960000294
Gulati, S., \& Gulati, A. (2012). Pulmonary manifestations of leptospirosis. Lung India: Official Organ of Indian $\begin{array}{lll}\text { Chest } & \text { Society, 29(4), } & \end{array}$ https://doi.org/10.4103/0970-2113.102822

Mehta, V., Bhasi, A., Panda, P. K., \& Gupta, P. (2019). A coinfection of severe leptospirosis and scrub typhus in Indian Himalayas. Journal of Family Medicine and Primary Care, 8(10), 3416-3418. https://doi.org/10.4103/jfmpc.jfmpc_614_19

MoHFW. (2021). Ministry of Health and Family Welfare | Home [Internet]. https://www.mohfw.gov.in/

PPCL. (2021). National Guidelines: Diagnosis, Case Management Prevention and Control of Leptospirosis. Programme for Prevention and Control of Leptospirosis. https://ncdc.gov.in/WriteReadData/1892s/File558.pdf

Roshanravan, N., Seif, F., Ostadrahimi, A., Pouraghaei, M., \& Ghaffari, S. (2020). Targeting cytokine storm to manage patients with COVID-19: a mini-review. Archives of Medical Research. https://doi.org/10.1016/j.arcmed.2020.06.012 